\title{
A mixed methods evaluation of a 4-week geriatrics curriculum in strengthening knowledge and comfort among orthopaedic surgery residents
}

Adrian C H Chan ${ }^{1,2^{*}}$, Victoria Chuen ${ }^{1,3}$, Andrew Perrella ${ }^{1,3}$, Guillaume Limfat ${ }^{4,5}$, Karen $\mathrm{Ng}^{1,6}$ and Vicky Chau ${ }^{1,6}$

\begin{abstract}
Background: In 2014, the University of Toronto Faculty of Medicine implemented a 4-week "Orthogeriatrics" rotation for orthopaedic surgery residents. We sought to assess the rotation's impact on trainees' knowledge, attitudes, and behaviours toward caring for older adults, and explore areas for improvement.
\end{abstract}

Methods: We used a mixed methods concurrent triangulation design. The Geriatrics Clinical Decision-Making Assessment (GCDMA) and Geriatric Attitudes Scale (GAS) compared knowledge, attitudes, and behaviours between trainees who were or were not exposed to the curriculum. Rotation evaluations and semi-structured interviews with trainees and key informants explored learning experiences and the curriculum's impact on resident physician growth and development in geriatric competencies.

Results: Among trainees who completed the GCDMA $(n=19)$, those exposed to the rotation scored higher in knowledge compared to the unexposed cohort (14.4 \pm 2.1 vs. $11.3 \pm 2.0, p<0.01)$. The following themes emerged from the qualitative analysis of 29 stakeholders: Increased awareness and comfort regarding geriatric medicine competencies, appreciation of the value of orthogeriatric collaboration, and suggestions for curriculum improvement.

Conclusions: These results suggest that the Orthogeriatrics curriculum strengthens knowledge, behaviour, and comfort towards caring for older adults. Our study aims to inform further curriculum development and facilitate dissemination of geriatric education in surgical training programs across Canada and the world.

Keywords: Orthogeriatrics, Orthopaedic surgery, Geriatric Medicine, Postgraduate medical education, Curriculum, Evaluation, Mixed methods

\footnotetext{
* Correspondence: adrian.chan@usask.ca

${ }^{1}$ Temerty Faculty of Medicine, University of Toronto, Toronto, Canada

${ }^{2}$ Department of Medicine, University of Saskatchewan College of Medicine,

Saskatoon, Canada

Full list of author information is available at the end of the article
}

(c) The Author(s). 2021 Open Access This article is licensed under a Creative Commons Attribution 4.0 International License, which permits use, sharing, adaptation, distribution and reproduction in any medium or format, as long as you give appropriate credit to the original author(s) and the source, provide a link to the Creative Commons licence, and indicate if changes were made. The images or other third party material in this article are included in the article's Creative Commons licence, unless indicated otherwise in a credit line to the material. If material is not included in the article's Creative Commons licence and your intended use is not permitted by statutory regulation or exceeds the permitted use, you will need to obtain permission directly from the copyright holder. To view a copy of this licence, visit http://creativecommons.org/licenses/by/4.0/. The Creative Commons Public Domain Dedication waiver (http://creativecommons.org/publicdomain/zero/1.0/) applies to the data made available in this article, unless otherwise stated in a credit line to the data. 


\section{Background}

Approximately $92 \%$ of hip fractures occur in adults aged 65 years and older [1]. Compared to other orthopaedic injuries, they are more strongly associated with mortality after one year,[2, 3] postoperative delirium,[4] loss of independence, $[5,6]$ and prolonged mobility limitations[2].

\section{Models of orthogeriatric care}

Orthogeriatric co-management care models were developed in the 1960's to address these poor outcomes [7]. Briefly, orthogeriatric care involves the interdisciplinary management of elderly patients with fragility fractures, [8-11] including geriatricians and specialized allied health teams on admission [12]. Literature comparing geriatric consult models with orthogeriatric ward-based care showed the latter was associated with reduced surgical wait times, [13] postoperative falls and complications, [14, 15] hospital length of stay, $[13,16,17]$ as well as shortand long-term mortality $[16,18]$.

Unfortunately, geriatricians in Canada remain scarce and concentrated in urban centers. Many older adults are therefore unable to receive the benefits of comanaged care [11]. In a recent survey, surgical trainees reported a lack of formal teaching and comfort involving perioperative management of older surgical patients [19].

\section{Geriatrics education in surgical training}

A handful of surgical residency programs have begun to develop and implement formal geriatric teaching to improve trainees' knowledge of caring for older patients. These range from didactic lectures to online discussions that seek to address learning objectives relevant to perioperative management such as delirium, pain management, falls, polypharmacy, and rehabilitation in the elderly population [20-22]. In general, knowledge and comfort in caring for older patients improved among residents who completed the course. Initiatives to formalize geriatric competencies are still ongoing, [23] yet little progress has been made in terms of establishing clear training and curricular objectives.

In 2014, the University of Toronto Department of Orthopaedic Surgery launched a 4-week Orthogeriatrics curriculum for postgraduate year 1 (PGY1) orthopaedic surgery residents throughout the year. Curricular components comprised: geriatric preoperative assessment, perioperative management of frail older adults with multimorbidity, inpatient and clinic-based geriatric assessments, and formal geriatrics educational seminars (Table 1).

This study sought to answer the following research questions:
Table 1 Orthogeriatrics curriculum components

\begin{tabular}{ll}
\hline Activity & Description \\
\hline Clinical & Inpatient \\
\cline { 2 - 2 } 2 weeks perioperative assessments: & - Orthopaedic elderly patients and management of \\
medically frail, complex older adults & 2 weeks inpatient geriatric consultation: \\
& $\cdot$ Comprehensive geriatric assessments on the \\
& Orthogeriatrics and surgical services \\
& Outpatient \\
& 1 day Perioperative Assessment Clinic \\
& $1 / 2$ day Falls Prevention Clinic \\
& $1 / 2$ day Geriatric Day Hospital Clinic \\
& $1 / 2$ day Geriatric Medicine Clinic \\
& Perioperative Teaching Rounds (Perioperative Risk \\
Educational & Assessment, Evidence Based Medicine, Perioperative \\
Content & Management e.g. Anticoagulation) \\
& Geriatric Giant Seminars (Dementia, Delirium, Falls, \\
& Incontinence, Constipation, Polypharmacy) \\
& Allied Health Seminars (Gait aids, Community Support \\
& Services, Capacity, Wound care) \\
& In-Training Evaluation Reports (ITERs) \\
& 360 feedback \\
& Exit interviews
\end{tabular}

1. What is the impact of the Orthogeriatrics curriculum on the residents' knowledge and attitudes pertaining to the management of elderly patients?

2. What are the areas of improvement within the current curriculum that will allow for further development?

\section{Methodology}

Our mixed methods concurrent triangulation design involved the simultaneous collection and analysis of quantitative and qualitative data (Fig. 1). We assessed residents using validated geriatric knowledge and attitude evaluations and converged these findings during data analysis with themes that emerged from semistructured interviews with the residents and key informants. The rationale for this approach was that corroboration between the two types of data would strengthen the validity of the program evaluation, and more robustly facilitate curriculum improvement.

\section{Study participants}

We collected data using a convenience sampling method from three participant groups: junior residents (PGY1-3) who had completed the rotation, senior residents (PGY4-5) who had not completed the rotation, and key informants (nurses, orthopaedic surgeons, and geriatricians) who worked closely with the residents.

\section{Quantitative data}

Junior and senior orthopaedic residents completed the University of Michigan Geriatrics Clinical Decision- 


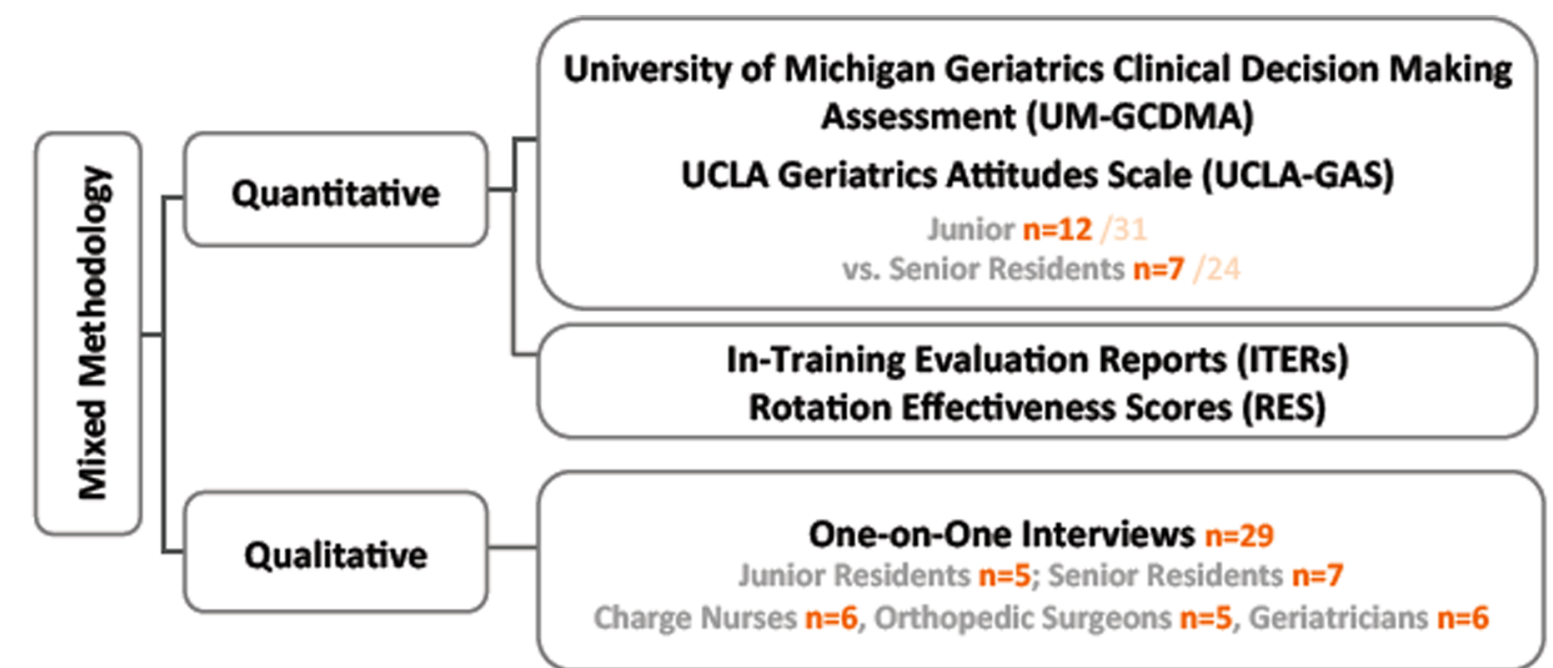

June 2017 Cohorts: "Senior Residents" (PGY4-5) no exposure to PGY1 Orthogeriatrics; "Junior Residents" (PGY1-3) exposed to PGY1 Orthogeriatrics

Fig. 1 Summary of the data collected using a mixed methods triangulation design

Making Assessment (GCDMA) and the University of California Los Angeles Geriatric Attitudes Scale (GAS) in June 2017. The GCDMA is a validated 20-item multiple-choice test which emphasizes inpatient care and common geriatric syndromes designed for medical and surgical residents [24]. The GAS is a 14-item Likert questionnaire developed to assess healthcare providers' attitudes towards caring for older patients [25, 26].

We also collected formal evaluations (In-Training Evaluation Reports [ITER] filled out by staff and Rotation Effectiveness Scores [RES] filled out by the junior residents) during the completion of their Orthogeriatrics rotation (Supplementary Files - ITER, RES).

\section{Qualitative data}

A research assistant [AC] conducted formal semistructured interviews with all three study participant groups (Supplementary File - Interview Questionnaires). Junior and senior trainees were asked about their opinions on geriatric education in the orthopaedic surgery residency program, as well as their feelings of preparedness in caring for the elderly. Likewise, key informants were asked about any perceived changes in the attitudes and practices of orthopaedic surgery residents as a result of the Orthogeriatrics rotation.

\section{Data analysis}

An independent-samples t-test was used to quantitatively compare the mean scores of knowledge and attitudes between junior and senior residents. Interviews were audio-recorded, transcribed verbatim, and organized using NVivo Software (Version 11.0). The data were qualitatively analyzed using grounded theory to develop a framework describing the Orthogeriatrics learning experience, its perceived impact on clinical competencies and attitudes towards caring for older adults, and areas for improved curricular performance [27]. A research assistant [AC] and PIs [VC, KN] independently read the transcripts and met periodically to refine the coding structure. This process continued until consensus was met and the coding structure was deemed stable. The research team then coded all transcripts and examined key themes that emerged. Qualitative analysis was performed concurrently with data collection to confirm that the interviews captured the information that we anticipated and concluded when theoretical saturation was achieved.

\section{Results}

Quantitative data

12 of 31 junior residents (39\%) and 7 of 24 (29\%) senior residents completed the GCDMA and GAS (see Table 2). We suspect that the low number of respondents was due to the voluntary nature of these assessments and that, although an effort was made to give residents protected time to complete them during their Academic Half Day (i.e., weekly teaching sessions), perhaps they had clinical duties that prevented them from participating.

\section{Knowledge and attitudes}

Junior residents scored statistically significantly higher on the GCDMA when compared to senior residents $(14.4 \pm 2.1$ vs. $11.3 \pm 2.0, p=0.009)$. No difference was found between the two groups on the GAS $(51.8 \pm 4.9$ vs. $51.7 \pm 8.1, p=0.979$ ). 
Table 2 Comparison of junior vs. senior resident scores on geriatric knowledge and attitudes

\begin{tabular}{lccc}
\hline & Number of Participants & GCDMA (mean \pm SD) /20 & $\begin{array}{l}\text { GAS (mean } \pm \text { SD) } \\
\text { /70 }\end{array}$ \\
\hline Junior Residents & 12 & $\mathbf{1 4 . 4} \pm 2.1$ & $51.8 \pm 4.9$ \\
Senior Residents & 7 & $\mathbf{1 1 . 3} \pm 2.0$ & $51.7 \pm 8.1$ \\
$p$-value & & $\mathbf{0 . 0 0 9 *}$ & 0.979 \\
\hline
\end{tabular}

GCDMA Geriatric Clinical Decision-Making Assessment; GAS Geriatric Attitudes Scale

\section{Rotation effectiveness scores}

Residents provided the following evaluations for the Orthogeriatrics rotation: 3.95 (2014-2015), 3.93 (20152016), and 4.25 (2016-2017). The scores, which are averaged out of 5 , assess the curriculum's overall organization, educational design, learning supports, climate, experience, and facilities (Supplementary File - RES).

\section{Qualitative data}

Interviews were conducted with junior residents (JR; $n=5$ ), senior residents (SR; $n=7)$, charge nurses $(\mathrm{CN} ; n=6)$, orthopaedic surgeons (OS; $n=5)$, and geriatricians $(\mathrm{G} ; n=6)$. The emerging themes are outlined below and in Table 3 :

\section{Awareness and comfort in geriatric competencies} Junior residents felt comfortable in their geriatric medicine competencies, particularly in the initial management of the geriatric giants. They were also able to extrapolate their knowledge to work through issues during a patient's hospital stay.

"I am comfortable in taking the first steps in getting them (older adults) optimized." (JR4).

"It really makes you feel more confident moving forward in terms of how to deal with these ward issues." (JR3).

In contrast, senior residents had mixed comfort in geriatric medicine competencies. Some felt their knowledge and skills were "probably a little limited" (SR5) and "would not be sufficient to provide a standard of care."

Table 3 Emerging themes from qualitative analysis of semistructured interviews

1. Awareness and comfort in geriatric medicine competencies

- A sense of increased comfort in various competencies involved in geriatric assessment and management, noted by both residents and nursing staff

2. Geriatric competencies strengthened by the curriculum:

- Sensitization to Holistic Care and Medical Complexity

- Communication with Older Adults

- Collaborative Relationships

3. Improving the Orthogeriatrics curriculum

- Suggestions for improvements in curricular design, such as preoperative management of elective cases, psychosocial dynamics with caregivers and families, and different pain management modalities
(SR6) Nursing staff also commented that senior residents were sometimes "not sure how to proceed because of all the medical complications." (CN1).

Geriatric competencies strengthened by the curriculum

Geriatric competencies strengthened by the curriculum Among the geriatric competencies, three sub-competencies appeared to be positively affected by the Orthogeriatrics curriculum.

1. Sensitization to Holistic Care \& Medical Complexity: Junior residents recognized the importance of comprehensive care, given the medical complexity of older surgical patients. As residents "learned to assess older adults more holistically", they began to see beyond the "mechanical" and "surgical" aspects of orthopaedic care (JR6). They also spoke of transferring their knowledge acquired from the geriatrics rotation to their surgical training:

"It's not just the surgery, it's the patient as a natural person that has [...] dementia or social problems. And now, when you get back into more surgical training, it's actually in your mind." (JR5).

2. Communicating with Older Adults: Junior residents also appeared to interact with older adults with improved bedside manner. Role-modelling appeared to play a significant role in developing these communications skills.

"Seeing how much time the internists and geriatricians spend really validates the fact that I might spend an extra bit of my time on the overnight call talking with them." (JR2).

Since the Orthogeriatrics curriculum, charge nurses observed "more conversation" (CN5) and "one-to-one patient time spent from the MDs." (CN6).

3. Collaborative Relationships: Understanding the role of the geriatrician and allied health professionals 
involved in patient care appeared to strengthen interprofessional appreciation among junior residents.

"It (Orthogeriatrics rotation) gives you more tools to know when to refer ... it gave me the potential to appreciate how important it is, and what to expect in a referral, and how to better prepare a patient before the geriatric team sees a patient." (JR5).

\section{Improving the orthogeriatrics curriculum}

The Orthogeriatrics rotation focused on managing older adults with emergent hip fractures. One suggestion was to teach about "perioperative assessments" (G2) for patients undergoing elective procedures.

"There should be an emphasis more on the pre-op assessment. So pre-ops for [...] all the elective cases - the elective knee replacements, hip replacements they probably need more teaching and experience around that." (G3).

In addition, disposition planning within complex psychosocial situations "to help deal with caregivers and family" (OS4) could be integrated more into the curriculum.

“... whatever setting orthopaedic residents are going to end up practicing in, that they have collaborative relationships [...] and make sure that their communication is open with the families and caregivers." (OS1).

\section{Discussion}

This research aims to further the current development of orthopaedic/geriatric care models and competences both in training and in practice [28-30].

Within clinical guidelines worldwide, orthogeriatric management for patients with a fragility fracture is the expected standard of care [31]. However, as echoed by our participants, a residents' scope of practice upon completion of their training remains highly variable, and resource limitations may limit a surgeon's access to a collaborative care model. Indeed, there were mixed opinions among senior orthopaedic residents who had not completed the Orthogeriatrics curriculum on whether they felt comfortable providing care to medically complex older patients. These sentiments are reflected in the literature among surgical faculty and residents, which further highlight the need for further geriatric training in medication, comorbidity, and delirium management [32].
Overall, the enhanced comfort and confidence in decision-making around older adult patients was noted by residents, surgeons, and charge nurses - especially as these were felt to be new traits among junior resident cohorts. The ability to transfer their learning from the curriculum to other services/rotations was of particular interest, as a prime concern was that such experiences in the PGY1 year would become lost amidst their rigorous surgical training. However, it appeared that the comfort with older adults the junior residents acquired has permeated longitudinally during their training thus far.

\section{Growth in knowledge}

Most striking for us was the apparent knowledge difference in geriatric competencies between junior and senior residents, as evidenced by the GCDMA scores. Although multiple competing factors could account for this difference (e.g. a focus on licensing examinations, operative skill, and independent practice during senior residency years), it was further corroborated by input from key informants that the junior cohort of residents appeared stronger in managing complex medical issues in the older adult patient.

Geriatric knowledge assessment among surgical residents appears to be a relatively new phenomenon. Acknowledging the difficulty in drawing comparisons given the paucity of studies using standardized scales, our junior resident mean score of $72 \%$ on the GCDMA is similar to that of geriatric fellows and senior internal medicine residents after their completion of a geriatricspalliative care rotation [26, 33]. A Dutch study designed a 6-week online course for faculty and residents involved in perioperative management of frail older patients [20]. Following the completion of these modules, the investigators noted an increase in knowledge and confidence scores among its participants. However, a limitation of this study is that it lacked a control group to compare with those who completed the online curriculum. Nonetheless, it may provide a reasonable alternative that is less resource-intensive and easier to implement across surgical residency programs.

\section{Growth in comfort}

Our interviews reinforced the positive impact of the curriculum on comfort in geriatric medicine competencies, and more specifically, the residents' appreciation for holistic and multidisciplinary care, as well as optimization of collaborative relationships and enhanced communication with older adults.

Another evaluation of a geriatric curriculum that used $16 \mathrm{~h}$-long didactic sessions to teach general surgery residents noted increased comfort in accessing community resources and multidisciplinary care to manage issues such as postoperative delirium and acute renal failure 
[21]. However, this study lacked objective testing in terms of knowledge base and therefore could not comment on whether there was a simultaneous improvement in the residents' understanding of patient management. While we and others did not find an objective impact on attitudes towards older adults on the GAS, our qualitative analysis demonstrated an overall embrace for the Orthogeriatrics curriculum from program stakeholders [26].

One should not discount the effect of role-modelling on geriatrics services in achieving holistic care. Several residents noted the opportunity to model their behaviour and patient communication based on their geriatric inpatient or perioperative hospitalist preceptors, as if being given permission to spend more time investigating a patient's concerns. Although we postulate that rarely in a surgeon's training would they be afforded time for lengthy consults, we believe that exposure to the culture of inpatient and outpatient geriatric care is what facilitated an appreciation for geriatric models of care, and sensitization to the older adult population.

That all participant groups applauded the necessity and applicability of Orthogeriatrics in residency training is a testament to both the growing complexity of patient care as well as gratification in being able to deliver such care.

\section{Sustainability of geriatrics curricula}

Several barriers in the design and implementation of a geriatric curriculum for residents were identified in the literature, [34] including limited geriatric faculty and services, an uncompromising curriculum, and lack of faculty or resident interest. Our educational program is sustained through existing orthogeriatric care models and geriatric clinical services, which fortunately have required minimal additional resources or administrative support.

Nonetheless, we discovered that the structure and content of our curriculum requires further improvement. With regards to curriculum structure, further discussions may help to optimize the balance between hospitalist and inpatient geriatric services that residents receive. There is also a need to broaden our clinical experiences to include topics like perioperative assessments of additional orthopaedic disorders and pain modalities in the perioperative setting. Furthermore, an understanding of discharge planning, especially in a post-fall hospitalization, is a critical aspect to resident training. Understandably so, as patients discharged to nursing homes have been shown to have higher readmission rates and prolonged lengths of stay [35, 36]. Current co-management orthogeriatric care models pay little emphasis to resident or surgeon education on discharge planning, and thus addressing this gap would be instrumental in improving our curriculum.

\section{Study limitations}

This study had several limitations from a research methodology perspective. First, it was challenging to recruit an adequate number of orthopaedic surgery residents. As such, the statistical comparison of geriatrics knowledge and attitudes between cohorts may not be statistically robust. Secondly, the University of Michigan GCDMA as an assessment of geriatric knowledge has been criticized for being outdated and concentrated on inpatient medicine [37, 38]. However, we employed this tool as an indicator of the knowledge growth generated by our curriculum, rather than reliance on a final numerical score. Finally, although our study utilized a validated test to evaluate the effect of a curriculum on resident knowledge, it did not explore its impact on clinically significant outcomes (e.g. adverse patient events, such postoperative delirium, lengths-of-stay, or hospital readmissions).

\section{Conclusions}

Our comprehensive evaluation demonstrates that residents have increased knowledge and comfort with managing geriatric issues on the wards following the completion of the 4-week Orthogeriatrics curriculum. Future steps for this curriculum include integrating the suggestions identified in our evaluation study and expanding geriatrics education programs to other surgical residency programs. We hope that our pilot work may serve as a model to other medical institutions to ultimately build capacity for stronger surgical training towards providing care for older adult patients.

\section{Abbreviations}

CN: Charge nurse; G: Geriatrician; GAS: Geriatric attitudes scale;

GCDMA: Geriatrics clinical decision-making assessment; ITER: In-training evaluation report; JR: Junior resident; OS: Orthopaedic surgeon;

PGY: Postgraduate year; RES: Rotation effectiveness score; SD: Standard deviation; SR: Senior resident

\section{Supplementary information}

The online version contains supplementary material available at https://doi. org/10.1186/s12909-021-02716-6.

Additional file 1. ITER. Clean version of Orthogeriatrics ITER.

Additional file 2. RES. Clean version of Orthogeriatrics RES.

Additional file 3. Interview Questionnaires. List of interview questions for study participants.

\footnotetext{
Acknowledgements

We thank the Retired Teachers of Ontario Foundation for their generous grant, without which we would not have been able to expedite and disseminate the Orthogeriatrics curriculum. We would also like to thank the Savlov/Schmidt Scholars Program in Geriatrics for providing postgraduate and medical students with mentorship and research opportunities working with older adults. Finally, this important work would not be possible without the close partnership between the Sinai Health System and University Health Network Hospitals' Divisions of Geriatric Medicine and Orthopaedic Surgery. We thank Dr. Samir Sinha, Dr. Vicky Chau, Dr. Karen Ng, Dr. Peter Ferguson,
} 
and Dr. Dan Stojimirovic for their key role in the development of the Orthogeriatrics curriculum.

\section{Authors' contributions}

AC collected, analyzed, and interpreted the data, and was a major contributor in writing the manuscript. VC, AP, and GL were major contributors in designing the study and writing the manuscript. KN and VC were major contributors in designing the study, analyzing and interpreting the data, and writing the manuscript. All authors read and approved the final manuscript.

\section{Funding:}

In 2016, the RTO/ERO Foundation awarded \$24,655 to Mount Sinai Hospital and the University of Toronto to support aging research and the training of post-secondary students in geriatrics and gerontology. This generous grant was used to fund the project as well as its research trainees' conferencerelated registration and travel fees.

\section{Availability of data and materials}

The datasets used and analyzed during the current study are available from the corresponding author on reasonable request

\section{Declarations}

\section{Ethics approval and consent to participate}

Ethics approval for the study was granted through the University of Toronto Research Ethics Board (protocol \#33469).

All study participants signed a consent form for the collection, analysis, and publication of their data during the time of recruitment. These forms are available from the corresponding author on reasonable request.

\section{Consent for publication}

Not applicable.

\section{Competing interests}

The authors declare that they have no competing interests.

\section{Author details}

${ }^{1}$ Temerty Faculty of Medicine, University of Toronto, Toronto, Canada. ${ }^{2}$ Department of Medicine, University of Saskatchewan College of Medicine, Saskatoon, Canada. ${ }^{3}$ Faculty of Health Sciences, Department of Medicine, McMaster University, Hamilton, Canada. ${ }^{4}$ Queen's University School of Medicine, Kingston, Canada. ${ }^{5}$ Department of Medicine, Western University Schulich School of Medicine \& Dentistry, London, Canada. ${ }^{6}$ Department of Medicine, Division of Geriatrics, Sinai Health System, Toronto, Canada.

\section{Received: 13 August 2020 Accepted: 5 May 2021}

\section{Published online: 17 May 2021}

\section{References}

1. Pillai A, Eranki V, Shenoy $R$, Hadidi M. Age related incidence and early outcomes of hip fractures: a prospective cohort study of 1177 patients. J Orthop Surg Res. 2011;6(1):5.

2. Prestmo A, Hagen $G$, Sletvold O, Helbostad JL, Thingstad P, Taraldsen $K$ Lydersen S, Halsteinli V, Saltnes T, Lamb SE, Johnsen LG. Comprehensive geriatric care for patients with hip fractures: a prospective, randomised, controlled trial. Lancet. 2015;25(9978):1623-33.

3. Leibson CL, Tosteson AN, Gabriel SE, Ransom JE, Melton LJ III. Mortality, disability, and nursing home use for persons with and without hip fracture: a population-based study. J Am Geriatr Soc. 2002 Oct:50(10):1644-50.

4. Bruce AJ, Ritchie CW, Blizard R, Lai R, Raven P. The incidence of delirium associated with orthopedic surgery: a meta-analytic review. Int Psychogeriatr. 2007 Apr;19(2):197-214.

5. Braithwaite RS, Col NF, Wong JB. Estimating hip fracture morbidity, mortality and costs. J Am Geriatr Soc. 2003 Mar:51(3):364-70.

6. Schnell S, Friedman SM, Mendelson DA, Bingham KW, Kates SL. The 1-year mortality of patients treated in a hip fracture program for elders. Geriatr Orthop Surg Rehabil. 2010;1(1):6-14

7. Devas MB, Irvine RE. The geriatric orthopaedic unit. A method of achieving return to independence in the elderly patient. Brit J Geriatr Prac. 1969;6:19-25.

8. British Orthopaedic Association. The care of patients with fragility fracture. London: British Orthopaedic Association. 2007 Sep:8-11.
9. Sabharwal S, Wilson $\mathrm{H}$. Orthogeriatrics in the management of frail older patients with a fragility fracture. Osteoporosis International. 2015 Oct 1; 26(10):2387-99.

10. Cunningham M, Kates S, Blauth M. Using a commitment to change tool for evaluation and planning of a global competency-based curriculum in orthogeriatrics. J Continuing Educ Health Prof. 2014;34(2):123-30.

11. Abrahamsen C, Nørgaard B, Draborg E, Nielsen D. Reflections on two years after establishing an orthogeriatric unit: a focus group study of healthcare professionals' expectations and experiences. BMC Health Serv Res. 2017 Dec 17(1):602.

12. Folbert EC, Hegeman JH, Vermeer M, Regtuijt EM, van der Velde TD, Ten Duis HJ, Slaets JP. Improved 1-year mortality in elderly patients with a hip fracture following integrated orthogeriatric treatment. Osteoporosis International. 2017 Jan 1;28(1):269 - 77.

13. Gupta A. The effectiveness of geriatrician-led comprehensive hip fracture collaborative care in a new acute hip unit based in a general hospital setting in the UK. JR Coll Physicians Edinb. 2014 Jan 1:44(1):20 - 6.

14. Friedman SM, Mendelson DA, Bingham KW, Kates SL. Impact of a comanaged Geriatric Fracture Center on short-term hip fracture outcomes. Archives of Internal Medicine. 2009 Oct 12;169(18):1712-7.

15. Stenvall M, Olofsson B, Lundström M, Englund U, Borssén B, Svensson O, Nyberg L, Gustafson Y. A multidisciplinary, multifactorial intervention program reduces postoperative falls and injuries after femoral neck fracture. Osteoporosis International. 2007 Feb 1;18(2):167 - 75

16. Grigoryan KV, Javedan H, Rudolph JL. Ortho-geriatric care models and outcomes in hip fracture patients: a systematic review and meta-analysis. Orthop Trauma. 2014 Mar;28(3):e49.

17. Khasraghi FA, Christmas C, Lee EJ, Mears SC, Wenz SJ. Effectiveness of a multidisciplinary team approach to hip fracture management. J Surg Orthop Adv. 2005:14(1):27-31.

18. Pajulammi HM, Pihlajamäki HK, Luukkaala TH, Jousmäki JJ, Jokipii PH, Nuotio MS. The effect of an in-hospital comprehensive geriatric assessment on short-term mortality during orthogeriatric hip fracture program - which patients benefit the most? Geriatric Orthopaedic Surgery Rehabilitation. 2017 Dec;8(4):183-91.

19. Shipway DJ, Partridge JS, Foxton CR, Modarai B, Gossage JA, Challacombe BJ, Marx C, Dhesi JK. Do surgical trainees believe they are adequately trained to manage the ageing population? A UK survey of knowledge and beliefs in surgical trainees. Journal of Surgical Education. 2015 Jul 1;72(4): 641-7.

20. Ozturk E, van lersel M, van Loon K, den Rooijen C, van Dongen E, van Wijngaarden RD, Klaase J, van Goor H. Interactive online learning on perioperative management of elderly patients. The American Journal of Surgery. 2018 Sep 1;216(3):624-9.

21. Barbas AS, Haney JC, Henry BV, Heflin MT, Lagoo SA. Development and implementation of a formalized geriatric surgery curriculum for general surgery residents. Gerontology geriatrics education. 2014 Oct:35(4)(2):380-94

22. Webb TP, Duthie E Jr. Geriatrics for surgeons: infusing life into an aging subject. Journal of surgical education. 2008 Mar 1:65(2):91 - 4.

23. Miller $M$, Rosenthal RA. Meeting the need for training in geriatrics: the geriatrics education for specialty residents program. J Am Geriatr Soc. 2017 Oct;65(10):e142-5.

24. Williams BC, Fitzgerald JT. Brief report: brief instrument to assess geriatrics knowledge of surgical and medical subspecialty house officers. J Gen Intern Med. 2006 May;21(5):490-3.

25. Reuben DB, Lee M, Davis JW Jr, Eslami MS, Osterweil DG, Melchiore S, Weintraub NT. Development and validation of a geriatrics attitudes scale for primary care residents. J Am Geriatr Soc. 1998 Nov;46(11):1425-30.

26. Ahmed NN, Farnie M, Dyer CB. The effect of geriatric and palliative medicine education on the knowledge and attitudes of internal medicine residents. J Am Geriatr Soc. 2011 Jan;59(1):143-7

27. Charmaz K. Constructing grounded theory: A practical guide through qualitative analysis. 13: Sage; 2006 Jan.

28. Quatman CE, Wiseman J, Phieffer L. Academic geriatric orthopedics: a new paradigm for inpatient care. Curr Geriatr Rep 2018;7(4):272-7.

29. Cunningham M, Kates S, Blauth M. Using a commitment to change tool for evaluation and planning of a global competency-based curriculum in orthogeriatrics. J Contin Educ Health Prof. 2014:34(2):123-30.

30. Grigoryan KV, Javedan H, Rudolph JL. Ortho-geriatric care models and outcomes in hip fracture patients: a systematic review and meta-analysis. Orthop Trauma. 2014;28(3):e49. 
31. Ftouh S, Morga A, Swift C. Management of hip fracture in adults: Summary of NICE guidance. BMJ. 2011 Jun 21;342.

32. Petronovich J, Wade TJ, Denson K, Webb TP. Elderly surgical patients: Are there gaps in residency education? Journal of Surgical Education. 2014 Nov 1;71(6):825-8.

33. Kishimoto M, Nagoshi M, Williams S, Masaki KH, Blanchette PL. Knowledge and attitudes about geriatrics of medical students, internal medicine residents, and geriatric medicine fellows. J Am Geriatr Soc. 2005 Jan;53(1): 99-102.

34. Gazewood JD, Vanderhoff B, Ackermann R, Cefalu C. Geriatrics in family practice residency education: an unmet challenge. Fam Med. 2003 Jan; 35(1)(1):30-4.

35. Riggs RV, Roberts PS, Aronow H, Younan T. Joint replacement and hip fracture readmission rates: Impact of discharge destination. PM\&R. 2010 Sep 1:2(9):806-10.

36. Deakin DE, Wenn RT, Moran CG. Factors influencing discharge location following hip fracture. Injury. 2008 Feb 1;39(2):213-8.

37. Siegler EL, Jalali C, Finkelstein E, Ramsaroop S, Ouchida K, Carmen TD, Logio $\mathrm{L}$. Assessing effectiveness of a geriatrics rotation for second-year internal medicine residents. J Graduate Med Educ. 2014;6(3):521-5.

38. Kalender-Rich JL, Mahnken JD, Dong L, Paolo AM, Hayley DC, Rigler SK. Development of an ambulatory geriatrics knowledge examination for internal medicine residents. J Graduate Med Educ. 2013;5(4):678-80.

\section{Publisher's Note}

Springer Nature remains neutral with regard to jurisdictional claims in published maps and institutional affiliations.

Ready to submit your research? Choose BMC and benefit from:

- fast, convenient online submission

- thorough peer review by experienced researchers in your field

- rapid publication on acceptance

- support for research data, including large and complex data types

- gold Open Access which fosters wider collaboration and increased citations

- maximum visibility for your research: over $100 \mathrm{M}$ website views per year

At BMC, research is always in progress.

Learn more biomedcentral.com/submissions 